\title{
Der Herrscher im Tempel \\ Bild und Inszenierung im kaiserzeitlichen Rom
}

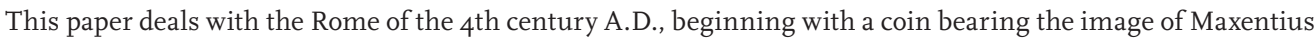
that depicts the emperor interacting with a personification of the Goddess Roma in her temple. It examines imperial strategies for self-depiction as a divine personage. Even if the depiction of the globe being passed from Roma to the emperor does not represent an actual event, it nevertheless refers to ceremonial acts situated in a specific architectonic context. The temple was restored by Maxentius and served, together with an imperial entrance hall (the Basilica of Maxentius) erected across from it, as a stage for an elaborate municipal and imperial ceremony. The coin image addresses these events, but, true to the nature of the medium, it exercises its ideological expressiveness without actually being present at the site of the ceremony. Construed in this way, the spatial references thus recall the modern phenomenon of cyberspace.

\section{Einführung}

Die Bezüge zwischen Bildwerken und Handlungen sind in allen Kulturen und zu allen Zeiten vielfältig. Statuen oder Reliefs können Handlungsabläufe beeinflussen, indem sie den Teilnehmern Hinweise oder sogar Anweisungen für das Verhalten übermitteln. Sie können auch selbst Handlungen darstellen und diese damit zeitlich fixieren und überhöhen. Der vorliegende Tagungsband bietet mit den Befunden in Knossos und in den mykenischen Palästen ebenso wie auf den syro-hethitischen Platzanlagen Beispiele dafür, wie solche Handlungswiedergaben ihrerseits dynamischer Bestandteil von Zeremonien werden können. Auch die klassische Antike kennt derartig eingebundene Bildwerke man denke an den Parthenonfries, der die Prozession der Panathenäen darstellte und sie zugleich räumlich begleitete, oder an die Friese des Titusbogens in Rom mit ihrer Wiedergabe eines Triumphzuges, wie er durch eben diesen Bogen in ähnlicher Form immer wieder geführt wurde. Der folgende Beitrag widmet sich einem nur scheinbar weniger offensichtlichen Fall der Verknüpfung von Handlung im Bild und in der Realität, zeitlich angesiedelt in der späten römischen Kaiserzeit. In den Jahren zwischen 307 bis $312 \mathrm{n}$. Chr. prägten die italischen Münzstätten mehrere Serien von folles mit dem Rückseitenbild des Venus- und Romatempels und der Umschrift CONSERVATOR VRBIS SVAE - Retter

Der vorliegende Text basiert auf den Studien des Verfassers für die 2006 an der Universität Hamburg abgeschlossene Dissertation »Das Rom des Maxentius«, die zurzeit in Druckvorbereitung ist. Für eine vertiefende Behandlung der hier angesprochenen Fragen sei auf diese Arbeit verwiesen. Vgl. auch Ziemssen 2007; Ziemssen 2010b; Ziemssen 2012. 

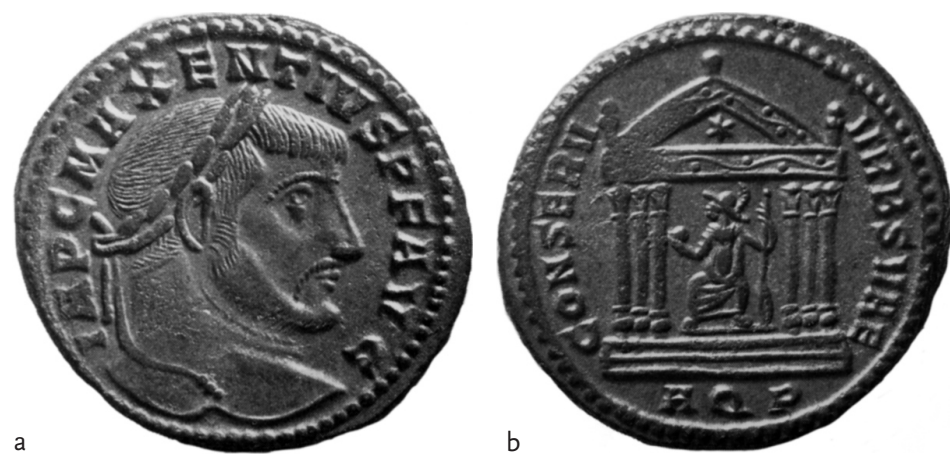

Abb. 1 | Follis, Aquileia, 307-310 n. Chr., max. Dm $26 \mathrm{~mm}$ a) Avers: Kaiser Maxentius, Umschrift IMP C MAXENTIVS P F AVG, b) Revers: Tempel der Venus und Roma mit Kultbild der Roma und Umschrift CONSERV - VRB SVAE (RIC VI Maxentius 121a).

seiner Stadt (Abb. 1) ${ }^{1}$. Auf den ersten Blick wirken diese Münzen im Rahmen der kaiserzeitlichen Prägung durchaus konventionell: Die Vorderseite gibt das Profil des zu dieser Zeit in Italien und der Provinz Africa regierenden Kaisers Maxentius wieder, während die Darstellung des Tempels auf der Rückseite, einer lang etablierten Ikonographie folgend, die Architektur schematisch beschreibt und in einem stark verbreiterten Mittelinterkolumnium das frontal thronende Kultbild der Göttin Roma mit dem Globus in der rechten Hand zeigt. Die Prägetradition dieser Darstellung von Tempel und Kultbild reicht in die severische Zeit zurück ${ }^{2}$. Bis in das spätere 3. Jh. hinein, vereinzelt auch noch in anderen Reichsteilen während der Herrschaft des Maxentius, wurde dieses Motiv durchgehend von der Münzlegende Roma(e) aeterna(e) begleitet und so eindeutig auf den Tempel der Göttinnen Venus felix und Roma aeterna nahe dem Forum Romanum bezogen ${ }^{3}$. Auf den Prägungen des Maxentius belegt noch die Statue der Gottheit selbst diese Identität. Der Tempel wird in schriftlichen Quellen nun allerdings zumeist als templum urbis bezeichnet und die Göttin Venus findet keine Erwähnung mehr .

Es sind zwei Aspekte, die die erneute Aufnahme dieses Münzmotivs im beginnenden 4. Jh. bemerkenswert machen und in das Zentrum einiger Überlegungen zu den Bezügen zwischen Handlungsformen und bildlichen Darstellungen in der späten Kaiserzeit treten lassen. Ungewöhnlich ist zunächst die Münzlegende conservator urbis suae, bei der es sich weder um eine be- noch umschreibende Ergänzung des Bildes zu handeln scheint.

$1 \quad$ Grundlage für die Analyse der maxentianischen Münzprägung ist der von C. H. V. Sutherland verfasste Band VI der Roman Imperial Coinage (RIC). Die Münzprägungen mit der Darstellung des Tempels und der Legende conservator urbis suae: RIC VI 271-277 (Ticinum); 305-309 (Aquileia); 338-347 (Roma); $393-397$ (Ostia). In Karthago werden Münzen mit der Legende conservator Karthaginis suae geprägt: RIC VI 417-435 (Carthago).

2 Einen Überblick über die Prägungen bietet Küthmann 1973, 34-38.

3 In großem Umfang war diese Kombination unter Probus (276-282 n. Chr.) geprägt worden (RIC V 2, 3738 Nr. 182-197; 62 Nr. 408-412; 96-97 737-741), außerdem unter Carausius und Allectus (286-297 n. Chr.: RIC V 2, 497 Nr. 389; 512 Nr. 578; 516 Nr. 613; 562 Nr. 40; 568 Nr. 113). Für Konstantin und Maximian erschien die alte Kombination noch im Jahr 307 n. Chr. (RIC VI 130 Nr. 99 (Konstantin). 100 (Maximian)), und für den afrikanischen Usurpator Alexander im Jahr 309/10 n. Chr. (RIC VI 434-435 Nr. 70. 75).

4 Zur spätantiken Geschichte des Tempels und der Namensgebung: Hausmann 2001, 193-194. 
Abb. 2 | Follis, Aquileia, 307-309 n. Chr., max. Dm $28 \mathrm{~mm}$, Revers: Variante der Darstellung in Abb. 1 b mit Maxentius im Interkolumnium des Tempels, Umschrift CONSERV - VRB SVAE (RIC VI Maxentius 113).

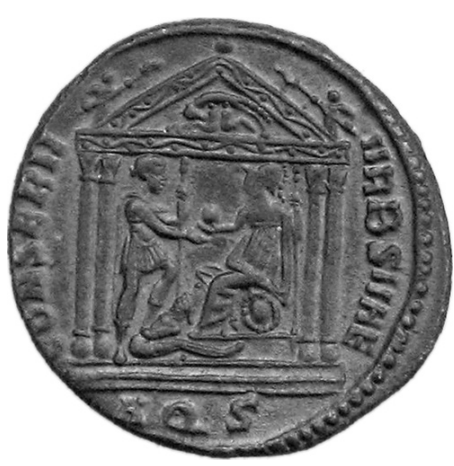

Offen deskriptiv war die frühere Legende Romae aeternae gewesen. Aber auch die unter Philippus Arabs zur Zeit der römischen Tausendjahrfeier im Jahr 248 n. Chr. zusammen mit dem Tempel verwendete Legende saeculum novum (neues Zeitalter) spielte noch in konnotativem Sinn auf die mit dem Bauwerk verbundene Ideologie des Neuanfangs und der aeternitas an ${ }^{5}$. Beim Ausdruck conservator urbis suae dagegen fehlt jeder offensichtliche Bezug. Es handelt sich dabei auch nicht, wie in der Forschung lange angenommen, um eine nüchterne Selbstbezeichnung des Maxentius als »Bewahrer« Roms und Erneuerer des Tempelbaues, der während seiner Herrschaft einem Feuer zum Opfer gefallen war $^{6}$. Eine semantische Analyse kann zeigen, dass mit der substantivischen Titulatur conservator vielmehr ein Reflex zeitgenössischer Akklamationen in der Münzprägung vorliegt, ähnlich wie mit dem Ausdruck expectate veni auf Münzen des Carausius oder dem bekannten redditor lucis aeternae auf dem Medaillon des Constantius Chlorus?. Ein neuer, expressiver und eng mit den Huldigungen der spätantiken Panegyrici verwandter Zug erfasste mit solchen Legenden seit dem späten 3. Jh. das zuvor wenig panegyrische Medium ${ }^{8}$. Der Begriff conservator verweist dabei unmittelbar auf zeremonielle Handlungen wie den adventus, bei dem ein Herrscher in der von ihm betretenen Stadt

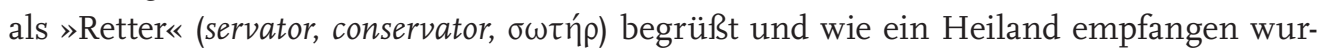
$\mathrm{de}^{9}$. Doch nicht nur mit dieser Legende lassen sich auf den maxentianischen Münzen

5 Gagé 1936, 170. 173-174; Körner 2002, 248-259.

6 Chronograph des Jahres 354 n. Chr. s. Maxentius imp. ann. VI: Hoc imp. templum Romae arsit et fabricatum est. Die Deutung des Maxentius als eines rückwärtsgewandten Anhängers der römischen Traditionen - eines »Bewahrers« - beherrscht die Forschung bis heute: vgl. insbesondere Cullhed 1994, 45.

7 Constantius Chlorus: Lehnen 1997, 80; Kolb 2001, 191-193; Carausius: Casey 1977, 222-223; Shiel 1977, 195; Casey 1994, 58. Zur Verbindung dieser Legenden mit der >Gefühlswelt< der Panegyrici vgl. MacCormack 1981, 29-31; Lehnen 1997, 81.

8 Zum adventus in der Zeit der Tetrarchie MacCormack 1981, 17-33; zur Übernahme von adventus-Akklamationen in die Münzprägung vgl. auch Lehnen 1997, 170.

9 Vgl. zu Verwendung und Deutung des Begriffes conservator seit der späten Republik Alföldi 1971; allgemein zu Akklamationen im Zusammenhang der adventus-Zeremonie Lehnen 1997, 169-170; zu bildlichen Darstellungen des adventus Hölscher 1967, 50-59; MacCormack 1981, 29-31. 
Momente repräsentativ überhöhter Handlung greifen. Der zweite ungewöhnliche Aspekt der Prägungen ist eine Bildvariante, die insbesondere von den Münzstätten Aquileia und Ticinum geprägt wurde (Abb. 2). In ihr tritt Maxentius selbst in das Bild und in den Tempel ein. Mit einem Fuß noch auf der Zugangsstufe, mit dem anderen aber fest auftretend bereits im Interkolumnium steht er der thronenden Göttin im Inneren des Tempels direkt gegenüber und greift mit der ausgestreckten Rechten nach ihrem Globus ${ }^{10}$. Erstmals in seiner über 100jährigen Geschichte wird das Bildmotiv des Tempels mit seiner frontalen Kultfigur damit variiert und regelrecht dynamisiert. Roma ist hier nicht mehr Kultbild, sondern interagierende Gottheit. An die Seite des Tempelinneren und ins Profil gerückt streckt sie den Globus dem Kaiser entgegen. Das auf römischen Münzen geläufige Bildmotiv »Tempel mit Kultfigur« wird zur Szene einer Handlung zwischen zwei Beteiligten. Dabei sind die räumlichen Angaben gerade in ihrer Schematik eindringlich, trennt doch die bildlich wiedergegebene Architektur in ein von der Kultfigur beherrschtes >Innen< und in die Außenwelt als den Bereich, aus dem der Kaiser kommt. Erst in dieser Variante der Tempeldarstellung wird auch die Legende mit der Anrufung des Kaisers unmittelbar verständlich. Die Bildvariation und die Legende verweisen gleichermaßen auf repräsentative Handlungen, auf Akklamationen, Einzugszeremonien und die Übertragung von Herrschaftslegitimation. Ungewöhnlich ist dabei ihre Verknüpfung und Ausdruckskraft, die mit den Darstellungskonventionen für den Venus- und Romatempel auf Münzen ebenso brechen wie mit den Gewohnheiten kaiserzeitlicher Ikonographie insgesamt: Im kaiserzeitlichen Bildbestand ist die Wiedergabe eines lebenden, handelnden Herrschers im Inneren eines Tempelbaues fast einmalig - wir werden dieses verblüffende Motiv weiter unten noch genauer untersuchen.

Die Bilder hatten ihren Platz in einem komplexen Bezugsrahmen von Politik, Architektur und Zeremoniell, der in seinen Grundzügen knapp skizziert sei ${ }^{11}$. Im Jahr 306 n. Chr. hatte Maxentius in Rom mit Unterstützung der Prätorianertruppen den Kaisertitel okkupiert. Er war zwar Sohn des Kaisers Maximian, hatte aber im tetrarchischen System keinen Herrscherposten erlangt. Nach seiner Usurpation und bis zur Niederlage gegen Konstantin im Jahr 312 n. Chr. stand Maxentius daher in einem wechselhaften Verhältnis zur Tetrarchie, bestimmt durch die erfolgreiche Abwehr von Angriffen in den Anfangsjahren, durch die zunächst gewährte und dann wieder entzogene Unterstützung seines Vaters sowie durch Bündnisse mit einzelnen Tetrarchen. Von Rom aus beherrschte er Italien mit den großen Inseln und, unterbrochen durch einen Aufstand, auch die Provinz Africa. Im dezentralisierten Reich war Maxentius der seit langer Zeit erste und zugleich der letzte Kaiser, der ohne Unterbrechung in der alten Hauptstadt residierte.

10 Eine der entsprechenden follis-Serien aus Aquileia ist laut RIC häufig belegt: RIC VI, 325 Nr. 113; das gleiche Motiv auf Nr. 114, allerdings im RIC mit »R« belegt. Zwei weitere Serien aus derselben Münzstätte sind bislang nur in Einzelexemplaren bekannt: Jelocnik 1973, 193-194 Nr. 92. 92a. Jeweils eine Serie aus Ticinum (RIC VI 296 Nr. 110) und Rom (RIC VI 378 Nr. 213) liegt in geringer Menge vor.

11 Zur Herrschaft des Maxentius vgl. Cullhed 1994 und zuletzt Ziemssen 2007, 12-34· 36-51. 
Noch einmal wurden hier nun die Mechanismen des Prinzipats mit dessen zeremoniell codierten Austausch zwischen dem Herrscher und den Gruppierungen der römischen Bevölkerung aktiviert. Dabei hatten sich die Voraussetzungen dieses alten »Akzeptanzsystems « (Flaig) seit der Soldatenkaiserzeit des 3. Jhs. und den Reformen der Tetrarchie verändert. Herrschaft wurde zu Beginn des 4. Jhs. unmittelbar göttlich begründet und entzog sich tendenziell der sozialen Kommunikation ${ }^{12}$. Das galt für die Herrscher der ersten und zweiten Tetrarchie nicht weniger als für deren Söhne Konstantin und Maxentius. Im Rom der Jahre zwischen 306 und 312 n. Chr. müssen daher die aus dem Prinzipat überkommenen Bindungsformen einerseits und die Repräsentation des Maxentius als eines entrückten Monarchen andererseits unmittelbar aufeinandergestoßen sein. Die Konsequenzen dieser außergewöhnlichen historischen Situation sind an den Großbauten jener Jahre abzulesen ${ }^{13}$. Sie entstanden in unmittelbarer Verbindung mit den kaiserlichen Palästen auf dem Palatin, sowohl im Süden als auch im Norden des Hügels. Im Süden näherte eine mit hohen Substruktionen bewältigte Erweiterung des Palastbereiches die Residenz dem Circus Maximus an, der zentralen >Begegnungsstätte < zwischen Herrscher und Bevölkerung. Zugleich verwandelten die Maxentiusbasilika und der Wiederaufbau des im Brand zerstörten Tempels der Venus und Roma (s. u. Abschnitt 5) das Gebiet nördlich des Palatin, das unmittelbar an den Haupteingangsbereich des Palastes angegliedert war. Die Basilika ist eine erweiterte Variante der zahlreichen zeitgenössischen Apsidensäle und konnte in ihrer monumentalen Gliederung breite Teile der Bevölkerung vor dem in der Apsis thronenden Herrscher aufnehmen. Der städtebauliche Bezug des Neubaus auf den gegenüberliegenden Tempel legt auch den Gedanken an Herrscherzeremonien in diesem Dreieck zwischen Palast, Aula und Tempel nahe, die das traditionelle Spektrum von Kaiserauftritten in Rom erweiterten und einen direkten Bezug zu den Göttinnen Venus und Roma herstellten: Auszüge des Kaisers aus dem Palast zum öffentlich zelebrierten Opfer vor den Fronten des Tempels, der Einzug in die Basilika, der Zug in das benachbarte Amphitheater oder den Circus Maximus.

\section{Handlungen im Bild: Globusübergabe und Opfer in der Darstellung der Münzen}

Diese im Architekturbefund erkennbare Intensivierung des städtischen Zeremoniells spiegelt sich - dies soll im Folgenden gezeigt werden - in den Bildern der zeitgenössischen Münzen wider. Eine methodisch grundlegende Bemerkung sei vorausgeschickt. Bilder und städtischer Raum sind mit den Handlungsformen einer Zeit stets eng verflochten. Dies gilt zunächst im generellen Sinne Bourdieus'; mit seinem Habitus-Begriff postuliert dieser die »allgemeine Disposition« einer Epoche, deren Äußerungsformen sich

12 Martin 1984; Flaig 1992, 174-207.

13 Vgl. Ziemssen 2007, 52-122; Ziemssen 2010b; Ziemssen 2012. 
auf allen Ebenen gesellschaftlicher Realität erkennen lassen ${ }^{14}$. Im Rom des beginnenden 4. Jhs. unterschied sich dieser gesellschaftliche Habitus wesentlich von jenem der ersten kaiserzeitlichen Jahrhunderte. Die mit plebs urbana, Senat und Soldaten ausgehandelte Akzeptanz des Maxentius in Rom beruhte auf dem skizzierten, im 3. Jh. gewandelten Herrscherbild und entwickelte sich vor dem Hintergrund einer zum seltenen Ereignis gewordenen kaiserlichen Präsenz in der Hauptstadt. Dieses neue Verständnis von Herrschaft kam in Zeremonien, Bauwerken und Bildern gleichermaßen zum Ausdruck. Zugleich können Bilder auch als unmittelbare Verweise auf spezifische, insbesondere zeremonielle Handlungen funktionieren. Vor dem Hintergrund dieser zweifachen Verknüpfung zwischen Bildern und der Gesellschaft, in der sie entstehen, werden die Abhängigkeiten von Raum, Handlung und bildlicher Darstellung im Folgenden analysiert. Die ereignishafte Szene der Globusübergabe und die akklamative Legende sprechen, das lässt sich bereits andeuten, für einen direkten Bezug des Bildes zum Kultgeschehen des templum urbis.

Die Frage, in welcher Art und Weise dieser Realitätsbezug auf den Münzbildern funktioniert, führt mitten in die Diskussion zum Verhältnis von »Symbolik« und »Wirklichkeit« der römischen Repräsentationskunst ${ }^{15}$. Es gibt keinen Zweifel, dass die Szene der Globusübergabe nicht im Sinne eines photographischen Realismus zu verstehen ist. Eine Globusübergabe durch einen Gott besaß im kaiserzeitlichen Zeremoniell keine unmittelbare Entsprechung. Ihre bildliche Formulierung wiederum ist durch Konventionen der Gattung und durch ideelle Konnotationen bestimmt. Vor allem seit der 2. Hälfte des 3. Jhs. zeigen zahlreiche Münzen die Globusübergabe durch eine Gottheit an den Kaiser ${ }^{16}$. Das Bildschema referiert auf die göttliche Unterstützung eines Herrschers und auf die Vorstellung des princeps a diis electus, die schließlich besonders im 3. Jh. und mit der Tetrarchie weite Verbreitung erfuhr. Die göttliche Wirkungsmacht erstreckte sich, folgt man vor allem den Aussagen der Panegyriker, unmittelbar auf das menschliche und herrscherliche Handeln ${ }^{17}$. Bilder der Globusübergabe auf Münzen sind dabei entsprechend ihrem Symbolcharakter in den meisten Fällen ohne einen räumlichen Kontext angegeben. Auch ein seltenes maxentianisches Goldmedaillon zeigt die Szene der Globusübergabe zwischen Roma und dem Kaiser in einer zur Bronzeprägung fast identischen Positionierung und Gewandung, jedoch ohne die Architektur des Tempels ${ }^{18}$. Die follis-Darstellungen könnten insofern zunächst als eine fast spielerische Innovation der römischen Münzpräger erscheinen, in der die beiden bekannten ikonographischen Formeln »Tempelbau« und »Globusübergabe« in einem einzigen Bild verschmelzen.

14 Vgl. die Definition von »Habitus« bei Bourdieu 1974, 144 als den »Ausdruck dieser allgemeinen Disposition [...], welche die Einzelmuster erzeugt, die sich dann in den verschiedenen Bereichen des Denkens und Handelns verwenden lassen «.

15 Vgl. Hölscher 1980.

16 Fears 1977, 279-299.

17 Martin 1984; Kolb 2001, 35-37.

18 RIC VI 373 Nr. 173; vgl. Carson 1980, 70 n. 110. 

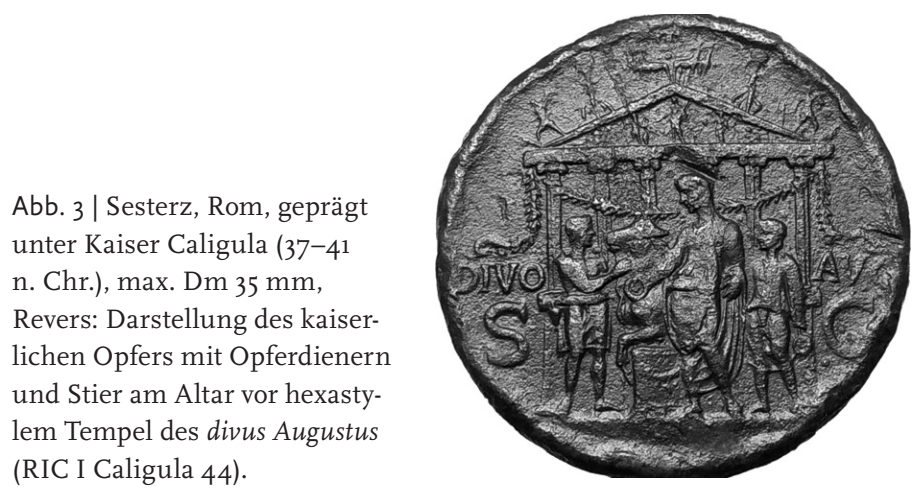

Doch dieser Eindruck trügt; um ein bloßes künstlerisches Experiment handelte es sich hier mit Sicherheit nicht. Dem römischen Betrachter war geläufig, dass in einem Münzbild wie auch in anderen staatlichen Bildwerken die Wiedergabe von Realität und deren symbolische Überhöhung zusammenfielen ${ }^{19}$. Semantisch vergleichbar aufgebaut sind etwa Werke der Staatskunst wie das Triumphrelief des Titusbogens, in dessen realistisch dargestelltem Zug wie selbstverständlich auch Gottheiten auftreten. Näher noch stehen der »Globusübergabe im Tempel« die aus Münzprägung und Reliefkunst bekannten Darstellungen kaiserlicher Opfer vor einem im Bild präzise identifizierbaren Tempelbau Tempelbau, etwa dem Münzbild des Caligula mit dem Opfer vor dem Tempel des divus Augustus (Abb. 3) ${ }^{20}$. Die vergleichbare Kombination von Tempelbau, handelndem Kaiser und Gottheit auf den maxentianischen Münzbildern konnte einen Betrachter durchaus an solche Opferszenen erinnern. Zwar sind auf den Opferdarstellungen die TempelKulisse und die vor ihr dargestellte Handlung gleichermaßen >real<, doch ist auch diese Wirklichkeit bereits inszeniert. Die Bilder geben ein Ritual wieder, das in der römischen Kaiserzeit durch die Platzierung des Altars in der Mittelachse des Tempels selbst »zum plakativen Bild der politischen Frömmigkeit, der pietas « wurde ${ }^{21}$. Eine Münzprägung des Alexander Severus zeigt nach diesem Bildschema den Kaiser beim Opfer vor dem durch die Legende ROMAE AETERNAE als das templum urbis identifizierten Heiligtum ${ }^{22}$. Das Thema dieser Darstellung ist eine Zeremonie zu Ehren der Roma und der Venus und damit der aeternitas Roms, wobei die Figur des Kaisers mit der von ihr verkörperten Tugend der pietas in den Mittelpunkt der Aussage rückt²3. Ähnlich sind Münzdarstellungen

19 Hölscher 1980.

20 Einen Überblick über Münzdarstellungen von Opfern vor Tempeln (mit zahlreichen Beispielen) bietet Scott Ryberg 1955, 186-189.

21 Hölscher 2006, 191-192.

22 Scott Ryberg 1955, 187-188 Abb. 115 a (mit dem Hinweis auf die Betonung der Kultstatue durch die Bildkomposition); Toynbee 1986, 103 Taf. 44, 4 .

23 Nach einer überzeugenden Hypothese Paul Zankers gilt diese Deutung auch für all jene Tempeldarstellungen, in denen nur die Kultstatue im Inneren sichtbar ist. Die Wiedergabe des Götterbildes verweise demnach 


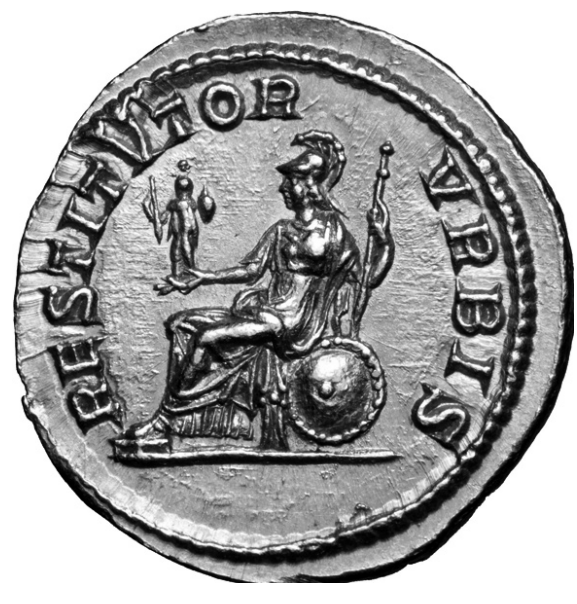

Abb. $4 \mid$ Aureus des Septimius Severus, Rom,207 n. Chr., max. Dm 21 mm, Revers: Thronende Göttin Roma, Umschrift: RESTITVTOR VRBIS (RIC IVa Septimius Severus 288; Hill 1964, 35 Nr. 975-977).

severischer Zeit zu deuten, die zwar nicht den Tempelbau einschließen, den maxentianischen Prägungen aber in anderer Hinsicht stark ähneln. Unter Septimius Severus erschienen mehrere Serien mit der Legende RESTITVTOR VRBIS, die zumeist den Kaiser beim Opfer zeigten ${ }^{24}$. Auf den ersten Serien wurde er allein vor einem Dreifuß wiedergegeben, in späteren Serien trat die thronende Figur der Roma auf der anderen Seite des Dreifußes auf. Schließlich erschien die Göttin in den spätesten Serien allein und ohne Dreifuß, aber weiterhin unter der alten, auf den Kaiser bezogenen restitutor urbis-Legende (Abb. 4). Die im Opfer zum Ausdruck kommende pietas gegenüber den Göttern trägt mit dem Verweis auf den Kaiser als Wiederhersteller (restitutor) der Stadt eine besondere Konnotation, die eng mit dem Begriff des conservator verwandt ist. Das substantivierte Verb bezeichnet auch hier den Kaiser als handelnden, Schutz und Fürsorge für Rom gewährenden Herrscher. Die restitutio urbis verwies einerseits auf Baumaßnahmen in Rom, andererseits aber auch auf die in diesen Aktivitäten zum Ausdruck kommende Erneuerung des Staates und die segensreiche Wirkung der severischen Dynastie für das Reich. Im Opfer vor Roma und in dessen Darstellung auf den Münzen verbinden sich diese Gedanken mit dem Konzept der pietas vor der Göttin der Stadt. Auch das reale Handeln und seine bildliche Wiedergabe sind insofern stets symbolisch überhöht.

stets auf den Anlass, bei dem es einem größeren Publikum auch real sichtbar wurde, also auf die kultische Zeremonie (Zanker 1997).

24 Zur Chronologie dieser Prägungen: Hill 1964. Zum Kontext vgl. Desnier 1993, 612-615; Daguet-Gagey 2004. 


\section{Handlungsformen: Der Kaiser im Kult}

Zurück zu den Prägungen des Maxentius. Sie ähneln den beschriebenen Darstellungen des herrscherlichen Opfers in ihrer Kombination von Kultbau und Kaiserfigur, unterscheiden sich aber auch in mehreren wesentlichen Aspekten von ihnen. Im Mittelpunkt der Szenen mit der Globusübergabe im Tempel steht nicht das Opfer, nicht die Idee religiöser pietas, sondern laut der Legende jene einer Auszeichnung des Herrschers als »Retter« seiner Stadt. Die conservatio urbis durch Maxentius ist Voraussetzung für die Verleihung der Herrschaft durch Roma, die hier somit auch die urbs conservata, die gerettete Stadt, repräsentiert. Während Prägungen wie jene des restitutor urbis Septimius Severus über die Opferdarstellung auf die menschliche Handlungssphäre des Kaisers verweisen, stellen die Münzen des Maxentius damit einen göttlichen, der Realität eines für alle Bürger sichtbaren Zeremoniells gerade enthobenen Handlungszusammenhang her. Fundamental unterschiedlich ist daher auch die Positionierung des Kaisers im Bild. In den Opferdarstellungen wie jener des Alexander Severus steht er stets vor dem Tempel am Altar, während das Tempelinnere als Ort der Kultfigur aufgefasst wird. Damit ist eine kultisch wichtige Trennung repräsentiert, die sich im Moment des Zeremoniells ebenso klar vor den Augen der Zuschauer manifestierte. Das Handlungsbild des Maxentius missachtet diese Dichotomie und nähert den Kaiser in seiner aktiven Schrittstellung unmittelbar der Gottheit an. Die Darstellung eines lebenden, handelnden Kaisers im Tempel ist in der Bildkunst außergewöhnlich und wird auf den zeitgenössischen Betrachter entsprechend drastisch gewirkt haben. Im Vergleich mit den Opferdarstellungen stellt sich hier rasch die Frage: War der Kaiser auch in der Realität im Inneren des Tempels gegenwärtig, und wenn ja, in welchem zeremoniellen Rahmen? Durchsucht man die Münz- und Reliefkunst der Kaiserzeit nach Wiedergaben von Kaisern in Tempeln, lassen sich tatsächlich fast ausschließlich Bilder von Kultstatuen eines Herrschers in Tempeln des provinzialen Kaiserkultes anführen, die sich mit der maxentianischen Handlungsszene nicht direkt vergleichen lassen - auch wenn sie die symbolische Bedeutung unterstreichen, die der Darstellung eines Kaisers im Inneren eines Tempels zukommen musste ${ }^{25}$. Es gibt allerdings eine Ausnahme, die für das Verständnis des maxentianischen Bildes aufschlussreich sein kann und auf der ebenfalls ein lebender Kaiser im Inneren eines Tempels bei der Interaktion

25 Tempel mit kaiserlicher Kultfigur: BMCRE Cilicia 117 n. 9; 118 n. 10; Price - Trell 1977, 276 n. 640 (Cilicia unter Maximinus Thrax); BMCRE Phrygia 307 n. 185 (munizipaler Kaiserkulttempel unter Domitian); BMCRE Pontus 105 n. 9-11. 108 n. 32; vgl. Hänlein-Schäfer 1985, 83-84 (Tempel der Roma und des Augustus in Nicomedia, manchmal mit der Figur des Kaisers allein, zuweilen auch mit bekränzender Roma); BMCRE Mysia 137 n. 236; 139 n. 242-245; 140 n. 253-256; 141 n. 257; 142 n. 267; 66 n. 360-363 (Roma-Augustus-Tempel in Pergamon); BMCRE I 196 n. 228; II 94 n. 449; $35^{2}$ n. 254*; III 12 n. 79; III 146 n. 711; Hänlein-Schäfer 1985, 81-82 (kleinasiatischer Provinzialtempel der Roma und des Augustus mit Augustusfigur und bekränzender Roma); BMCRE Mysia 142 n. 262-266; Radt 1999, 209-212 (Avers mit Wiedergabe des kleinasiatischen Provinzialtempels, Revers zeigt das Traianeum mit Statuen des Zeus und Trajans). 


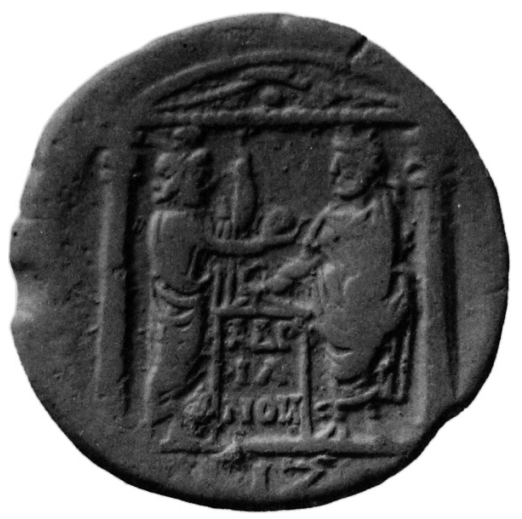

Abb. 5 | Billontetradrachme des

Hadrian, Alexandria, 132/33,

Revers: Tempel mit Hadrian

und Serapis im Interkolumni-

um (Milne 1933, n. 1380 pl. 4).

mit der Gottheit erscheint: Auf den Rückseiten hadrianischer Bronze-Münzserien aus Alexandria, die möglicherweise aus Anlass des kaiserlichen Besuchs in der Stadt im Jahr 132 entstanden, sind stets Hadrian und Serapis im Interkolumnium eines distylen Tempels dargestellt ${ }^{26}$. Für uns besonders aufschlussreich ist eine in das Jahr 132/133 n. Chr. datierende Serie, in deren Bild der links stehende, in der Linken ein Szepter haltende Serapis dem ihm rechts gegenüber dargestellten Hadrian den Globus überreicht (Abb. 5) ${ }^{27}$. Noch ein weiteres Exemplar zeigt den Gott bei der Übergabe eines Objektes an den Kaiser, in diesem Fall eines Kranzes mit Taenia ${ }^{28}$. Auf allen anderen Münzen dieser Serien erhebt Serapis den rechten Arm in Richtung auf den gegenüberstehenden Kaiser. Der Kaiser ist dabei in fast gleichbleibender Weise dargestellt. Er hält in seiner Linken zumeist ein Szepter und legt seine Rechte stets auf eine halb hohe, rechteckige und von einem Giebel bedeckte Stele, die zwischen den beiden Figuren aufgestellt ist und in ihrem Feld den Namen Hadrians im Akkusativ trägt ${ }^{29}$. Bei dem Tempel handelt es sich mit großer Wahrscheinlichkeit um den aus der Zeit Ptolemaios' III. (246-221 v. Chr.) stammenden Serapistempel auf dem Gelände des alexandrinischen Serapeions, dessen Grundmauern bei Ausgrabungen identifiziert werden konnten ${ }^{30}$. Der Bau wurde unter Trajan und Hadrian mehrfach auf alexandrinischen Münzen geprägt, stets aber nur mit dem Gott und dem als Inschriftenstele zu deutenden Gegenstand ${ }^{31}$. Auf diesen Bildern legt Sera-

26 Vogt 1924, 69-70; BMCRE Alexandria 101-102 n. 874-876; Tran tam Tinh 1983, 188-189 (Kat. IVB 42).

27 Aus der Sammlung Dattari (n. 1946). Vgl. Milne 1933, n. 1380 pl. 4; Tran tam Tinh 1983, 188 (Kat. IVB 42 c).

28 Tran Tam Tinh 1983, 133 (Kat. IC 31).

29 Zusammenfassend und mit älterer Literatur: Tran tam Tinh 1996, 223.

30 McKenzie - Gibson - Reyes 2004, 85-90.

31 Die Stele erscheint auch auf den früheren Darstellungen des Tempels (unter Trajan wie auch vor und nach der hier behandelten Serie unter Hadrian), auf denen der Kaiser noch fehlt, und trägt dort eine nur durch Punkte angedeutete Schrift, s. Tran tam Tinh 1983, 126-133 (Kat. IC 12-30); Handler 1971, 65-67. Vgl. Vogt 1924, 69-70. Unwahrscheinlich ist die gelegentlich vorgenommene Deutung der Stele als Naos und damit als Hinweis auf eine Baumaßnahme Hadrians im Serapeum (u. a. Beaujeu 1955, 230-231, offensichtlich auch Tran Tam Tinh 1996, 223). 
pis selbst seine Hand auf den Giebel und besetzt die Stele somit für sich ${ }^{32}$. Nur auf den hier behandelten Münzen Hadrians ist die Stele durch die Angabe des Kaisernamens im Akkusativ eindeutig als Widmung an Hadrian gekennzeichnet und deutet damit auf eine Ehrung des Kaisers im Tempel des Serapis hin ${ }^{33}$. Dass Hadrian im Bild auch selbst innerhalb des Tempels dargestellt ist und ihm die Stele durch den Gestus der Handauflegung in der gleichen Form zugeschrieben wird wie auf den früheren Münzbildern dem Serapis, lässt an eine Aufnahme des Kaisers in den Tempel denken. Auch der grüßende Gestus des Gottes deutet dies an ${ }^{34}$. Auszugehen ist zumindest von einer Statuenweihung, entsprechend den zahlreichen Widmungen an Hadrian, die für das athenische Olympieion überliefert sind ${ }^{35}$. Wie in Athen muss dies auch in Alexandria nicht zwangs-

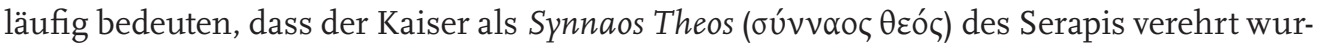
$\mathrm{de}^{36}$, wie dort aber ist anzunehmen, dass er im Tempel und dem umgebenden Kultbezirk in statuarisch prominenter Form vertreten war. Mit einiger Wahrscheinlichkeit bezogen sich die Serapis-Prägungen mit Hadrian im Tempel auf solche nicht mehr rekonstruierbaren Ehrenbeschlüsse für den Kaiser ${ }^{37}$. Entsprechend lässt sich auch die gelegentlich dargestellte Übergabe von Kranz oder Globus durch Serapis verstehen. Sie interpretiert den Bezug des Gottes zum Kaiser als göttliche Auszeichnung und Verleihung von Herrschaftsrecht und Sieghaftigkeit, analog zu der römischen Tradition, höchste Götter als $\sigma \omega \tau \eta ́ p \varepsilon \varsigma$ bzw. conservatores der Herrscher anzusehen und ihnen auch die Garantie der Herrschaft zuzuschreiben $^{38}$. Zwei hadrianische Bauinschriften von Serapistempeln an anderen Stellen Ägyptens bringen genau diese Aspekte zum Ausdruck, indem sie die entsprechenden

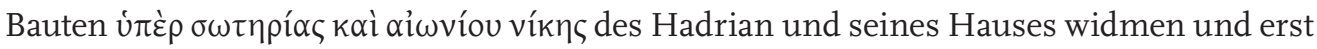
dann den Namen des Gottes nennen (Zeus Helios, der große Serapis) ${ }^{39}$.

32 Vogt 1924, 70 vermutet zu Recht eine auf Serapis bezogene Inschrift und denkt an die Verkündung eines von Serapis vollbrachten Wunders oder einer Weihung an den Gott.

33 Vgl. zur Form griechischer Ehreninschriften Guarducci 1974, 89-102. Handler 1971, 67-68 bemüht sich um eine genauere Erklärung der Szene, indem sie diese als Hinweis auf einen administrativen Akt Hadrians deutet. Die Stele würde demnach die öffentlichen Archive Alexandrias versinnbildlichen, die ihren Platz stets im Serapistempel unter dem Schutz des Gottes gehabt hatten und erst unter Hadrian in die von ihm neugeschaffene Bibliothek verlegt, also gewissermaßen unter seinen Schutz gestellt wurden. Abgesehen von der für eine Münzdarstellung wohl etwas zu komplexen politischen Rekonstruktion berücksichtigt diese Deutung nicht, dass die Darstellung des Kaisers im Tempel auf einer Münze außergewöhnlich ist und sich kaum befriedigend mit dem (gemutmaßten) Standort der Archivtafeln erklären lässt.

34 Beaujeu 1955, 230-231 deutet diesen Gestus als Zeichen für die Aufnahme Hadrians durch Serapis als oúvvoos $\theta \varepsilon o ́ \varsigma$.

35 Benjamin 1963, vgl. dort bes. 73-74 (Stele für Hadrian, nicht im Hadrianeum, geweiht durch zwei Phylen); Guarducci 1974, 92-93; Willers 1990, 48-62.

36 Vogt 1924 vermutet, die Stele verkünde die Aufnahme des Hadrian in den Tempel als oúvvoo $\theta \varepsilon o ́ \varsigma$. Hornbostel 1973, 382 deutet die seines Erachtens ebenbürtige Darstellung von Gott und Kaiser umstandslos als Beleg für eine solche Stellung Hadrians.

37 Tran tam Tinh 1996, 222.

38 Serapis galt wie Zeus-Jupiter als allumfassende Gottheit und wurde in der hadrianischen Religionspolitik in gleicher Weise mit dem Kaiser verbunden wie Zeus (Beaujeu 1955, 232-236; Tran tam Tinh 1996, 222-223).

39 Bernand 1977, 59 n. 21 (Architrav mit der Inschrift eines Tempels des Zeus Sol, des großen Serapis, gefun- 
Die hadrianischen Prägungen bieten somit selbst eine Erklärung für ihre ungewöhnliche Darstellung des Herrschers im Tempel. Kaiser und Gottheit sind hier Bestandteile einer Handlungsszene, die zwar nicht bildrealistisch ist, symbolisch aber dennoch unmittelbar mit einer materiellen Ehrung des Herrschers im Tempel verknüpft ist. Wie das Opfer auf den zuvor genannten Münzdarstellungen bezieht sich auch die Globusübergabe auf den hadrianischen Münzen auf eine spezifische Position des Kaisers im Tempel, doch rückt anstelle der pietas seine passive Teilhabe am Kult in den Mittelpunkt, konnotiert mit den Vorstellungen der Herrschaftslegitimation und der Sieghaftigkeit. So weit nun diese Darstellung zeitlich und geographisch auch von jener des Maxentius im Tempel der Roma entfernt ist, verbindet beide doch ein vergleichbarer politischer Kontext ${ }^{40}$. Die alexandrinische Münzprägung des Jahres 132/133 n. Chr. stand noch unter dem Eindruck des erst kurz zuvor abgeschlossenen, mehrmonatigen Aufenthalts Hadrians in der Stadt (130/131 n. Chr.). Der in eigener Person gegenwärtige Herrscher muss Gegenstand intensiver panegyrischer Bekundungen der Bevölkerung geworden sein; auf Münzen wurde sein adventus umfangreich gefeiert. Die Präsenz Hadrians könnte somit auch die entsprechenden Beschlüsse zu seiner Aufnahme in das zentrale Heiligtum des Serapis ausgelöst haben. Im Rom des beginnenden 4. Jh. ergab sich mit der erneuerten Präsenz eines Herrschers eine ähnliche Situation. Die Stadt feierte ihren seit Jahrzehnten ersten dauerhaft anwesenden Kaiser als conservator urbis. Damit verbanden sich ohne Zweifel panegyrische Huldigungen, wie wir sie für zeitgenössische Herrscher kennen, ebenso wie die in der gesamten Kaiserzeit üblichen Ehrenbeschlüsse des Senats. Eine Aufnahme des Maxentius in den Venus- und Romatempel ist in diesem Zusammenhang äußerst wahrscheinlich.

\section{Ideologische Bilder: Maxentius und die aeternitas}

Diese Verbindung zwischen Göttin und Kaiser lässt sich noch näher bestimmen. Das oben bereits erwähnte maxentianische Medaillon mit der isolierten Szene der Globusübergabe trägt die einzigartige Legende ROMAE AETERNAE AVCTRICI AVG N (Abb. 6) ${ }^{41}$. Dieser Begriff auctor (F. auctrix) - der Urheber, Begründer einer Sache - hatte im Verhältnis eines Gottes zu einem Menschen eine sehr spezifische Bedeutung. Roma Auctrix Augusti bezeichnete die Göttin nicht nur als Urheberin der Herrschaft (auctrix imperii). Der auctor eines Menschen war auch sein Schöpfer in einem genealogischen Sinne, sein

den in Gebel Dokhan, datiert auf 117-119 n. Chr.); 98 n. 42 (Architrav mit der Widmung eines Tempels des Zeus Sol, des großen Serapis und verbundener Gottheiten, gefunden in Gebel Fatireh (in situ), datiert auf den 23. April 118 n. Chr.). Vgl. auch Bernand 1984, 77-78 Kat. 16. 17.

40 Es lässt sich nicht völlig ausschließen, dass hier ein direkter Einfluss der alexandrinischen Münzprägung auf die stadtrömische vorliegt; doch spricht angesichts des zeitlichen Abstands wenig für eine solche Hypothese.

$41 \quad$ RIC VI 373 Nr. 173; vgl. Carson 1980, 70 n. 110. 

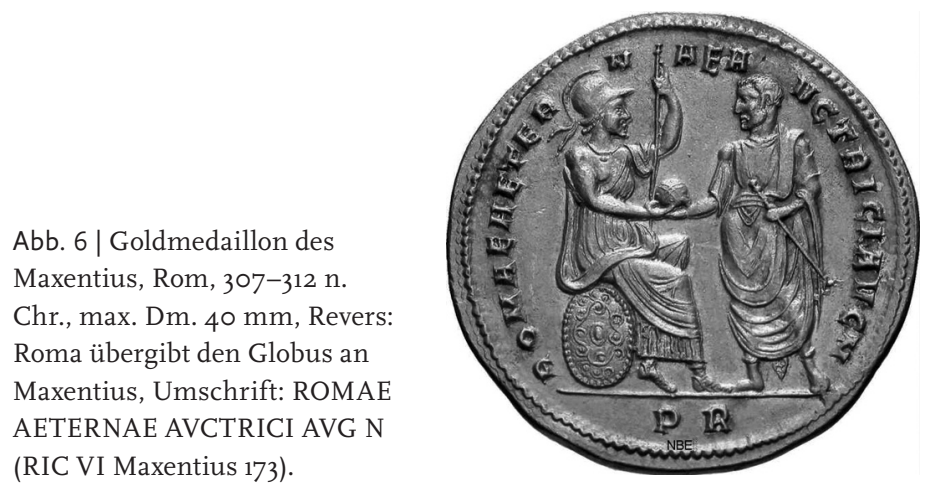

Ahnherr ${ }^{42}$. Dieses Verständnis eines Gottes ist für die Zeit der Tetrarchie gut bekannt; die Götter - Jupiter und Hercules - galten nicht nur als Beschützer der Kaiser und Garanten ihrer Herrschaft, sie waren darüber hinaus auch die Begründer der beiden kaiserlichen Familienzweige der Iovii und der Herculii ${ }^{43}$. Die göttliche Filiation der beiden Augusti war Grundlage des tetrarchischen Herrschaftssystems und im Panegyricus auf Maximian von $291 \mathrm{n}$. Chr. wird Jupiter auch als der auctor deus des Diocletian angesprochen, als der »Schöpfergott« und göttliche Vorvater des Kaisers ${ }^{44}$. Roma und Maxentius waren somit über einen komplexen und für die Zeit der Tetrarchie spezifischen ideologischen Diskurs miteinander verbunden. Roma rückt in der Medaillonprägung als Schöpferin von Person und Status des Maxentius an die Stelle der tetrarchischen Schutzgötter. Es ist anzunehmen, dass diese Rolle der dea Roma in der Kommunikation zwischen den neuen Herrschern und den stadtrömischen Gruppierungen sowie in den Huldigungsadressen der Festredner entstand und ihr kein systematischer Entwurf zugrundelag, wie es ihn offenbar für die Tetrarchie gab. Damit wurde die im hadrianischen Tempel kultisch eng definierte Roma aeterna zu einer Herrschaftsstifterin, die dem Maxentius nicht wie Jupiter oder Herkules den Tetrarchen in abstraktem Wesen gegenüberstand ${ }^{45}$; Maxentius wurde stattdessen unmittelbar - und exklusiv - mit dem örtlich klar definierten Kult der Roma im templum urbis assoziiert. Die Bronzeprägungen mit dem Bild des Tempels referieren auf diesen Bezug des Kaisers zum Kultbau im Stadtzentrum und wenden sich daher auch mit der Legende conservator urbis suae nicht ohne Grund immer an den Kaiser, auch wenn nur der Tempel mit der Kultfigur der Göttin, nicht der Kaiser dargestellt ist. Ähnlich wie die Münzen des Hadrian für den Tempel in Alexandria scheinen somit

\footnotetext{
42 TLL 4,2, 1204 Z. 58-59 s. v. auctor.

43 Kolb 2001, 35-37.

44 Pan. XI (3), 3, 4.

45 Jupiter, Herkules oder Mars verwiesen in Szenen der Globusübergabe nie auf einen spezifischen Kult, besaßen somit keine dem Kaiser physisch zugängliche Ausdrucksform und waren auch durch die häufige Legende CONSERVATOR AVGVSTI nur in der ihm gegenüber ausgeübten Schutzfunktion gekennzeichnet.
} 
auch die maxentianischen Prägungen eine Aufnahme des Kaisers in das templum urbis zu spiegeln, sei sie über die Weihung von Statuen oder im Zusammenhang des Neubaus über eine entsprechende Widmungsinschrift erfolgt. Für eine solche Rolle des Kaisers im Kultbau spricht auch eines der wenigen Zeugnisse der Geschichtsschreibung zu den Ereignissen jener Jahre. Aurelius Victor berichtet über die Tage nach der Niederlage des Maxentius, dass der Senat dem Sieger Konstantin das templum urbis, ebenso wie die Maxentiusbasilika, »geweiht « habe (Caes. 40, 6: sacraverunt), ihm den Bau also zusprach, wie es gewöhnlich für einen Gott geschieht. Mit großer Wahrscheinlichkeit wiederholte das Gremium damit nur einen Akt, den es bereits gegenüber dem ursprünglichen Bauherren Maxentius vollzogen hatte und der jeweils als Inschrift an prominenter Stelle verkündet worden sein muss.

Mit diesen Bezügen steht das maxentianische Bild der Globusübergabe im Kontext einer Zeit, die den direkten Einfluss der Götter auf das irdische Geschehen und auf die Handlungen der Herrscher annahm. Nicht mehr die pietas, die einem menschlichen Handlungsrahmen entstammt, sondern die Nähe zur göttlichen Natur war nun legitimationsstiftend ${ }^{4}$. Die zeitgenössische Panegyrik bietet dafür eine aufschlussreiche Parallele mit der berühmten Schilderung der Apollo-Vision des Konstantin47. Der Panegyriker baut nach der niedergeschlagenen Rebellion des Maximian ein neues, politisch von der Tetrarchie abgewandtes Herrscherbild Konstantins auf. Gegen Ende der Rede beschreibt er den Abstecher Konstantins zu einem Heiligtum - zu identifizieren vermutlich mit einem auch archäologisch nachgewiesenen Tempel des Apollo in den Vogesen. In diesem templum toto orbe pulcherrimum sieht der Kaiser - so beschreibt es der Redner, gemildert durch ein respektvolles credo - Apollinem tuum comitante Victoria coronas tibi laureas offerentem. Die Passage ist viel diskutiert und kann hier nicht in all ihren möglichen Bezügen beleuchtet werden. Für unseren Zusammenhang wichtig ist die Tatsache, dass die Annäherung von Kaiser und Gott auch hier als Handlung in einem Heiligtum verortet wird. Die Sprache der Münzen und der Panegyrici beweisen hier ihre enge Verwandtschaft, sowohl im sprachlichen Ausdruck, der die außergewöhnliche Natur dieser Nähe emphatisch hervorhebt (Apollo tuus/urbs sua), als auch in der Bildsymbolik. Die von Apollo und Victoria dargereichten Kränze sind als Zeichen für die Dauer eines Lebens oder einer Herrschaft von Münzdarstellungen her bekannt und die Schilderung erinnert insofern auch nicht zufällig an die Übergabe des Globus durch Roma an Maxentius. In beiden Fällen ist die Nähe zum Gott auch die Basis für das Herrschaftsrecht des Kaisers. Wenige Zeilen später spricht der Panegyriker von der Weltherrschaft des Apollo/Konstantin ${ }^{48}$. Konstantins Apollo ist ebenso wenig vom tetrarchischen Herrscherbild zu trennen wie die Roma des Maxentius: Stets betont die zeitgenössische Feier eine intime, exklusive

46 Martin 1984.

47 Pan. VI 21, 3-7. Vgl. zu dieser Schilderung Rodgers 1980, 259-278 sowie den Kommentar der Passage bei Nixon - Rodgers 1994, 248-251.

48 Pan. VI 21, 5 . 
Abb. 7| Tempel der Venus und Roma, westliche Cella des unter Maxentius restaurierten Bauwerks nach der neuzeitlichen Restaurierung der zoer Jahre des 20. Jh.

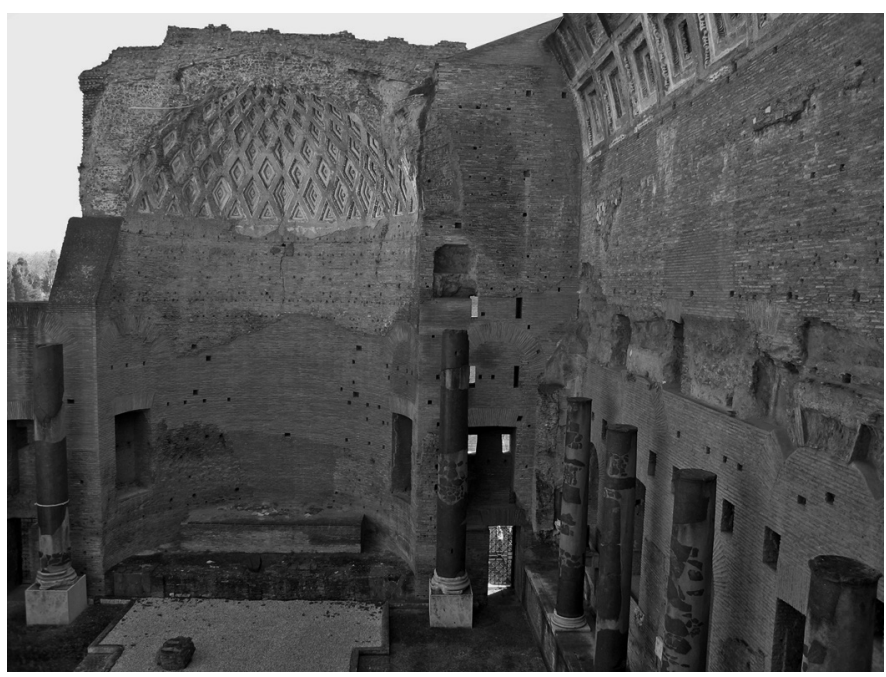

Nähe von Herrscher und Gottheit, die in der Verwandtschaftsbeziehung der Tetrarchen und ihrer Schutzgötter vorgebildet ist. Die Hinwendung zu den spezifischen Gottheiten Apollo und Roma, die in den Medien des Panegyricus und der Münzen sehr bewusst herausgestellt wurde, zeigt dabei aber zugleich die politische Abwendung vom System der Tetrarchie. In den Krisenjahren der Tetrarchie nach 305 waren solche religiösen Bezeugungen immer auch öffentlich verbreitete Akte, auf die neben den Herrschern selbst auch die Erwartungen der mit ihnen verbundenen Gruppen Einfluss nahmen. Die Panegyriker und die Münzprägestätten brachten Vorstellungen zum Ausdruck, die der Herrscher und seine Untertanen übereinstimmend teilen konnten 49 .

\section{Handlungen im städtischen Raum: Maxentius in Rom}

Zurück zur Stadt Rom. Die Münzprägungen mit der Globusübergabe durch Roma an Maxentius müssen auch hier Teil eines breiten, für uns allerdings vollständig verlorenen panegyrischen Diskurses gewesen sein. Sie sind zugleich - hierin vergleichbar den Münzdarstellungen mit einem kaiserlichen Opfer - räumlich präzise lokalisiert. Die Darstellung des Tempels der Venus und Roma verweist auf das reale Bauwerk, einen der größten Tempelbauten Roms, der noch dazu durch die zeitgleichen, aufwendigen Wiederherstellungsmaßnahmen den Zeitgenossen äußerst präsent sein musste (Abb. 7). Die Rolle der Roma gegenüber dem Kaiser ist insofern nicht allein im Sinne eines ideologischen

49 Zum Bezug zwischen der Apollo-Vision, der Hinwendung zum Christentum und der politischen Situation des Jahres 310 n. Chr. vgl. zuletzt Barceló 2007. 
Konzeptes zu verstehen, vielmehr bildete sie sich in unmittelbarem Zusammenhang mit der Erneuerung des Bauwerkes selbst heraus. Derartige Verbindungen zwischen der Konzeptionierung einer Herrschaft und ihrer architektonischen Formulierung lassen sich in Rom vielfach nachweisen. Zwei besonders aufschlussreiche Beispiele bieten Ereignisse aus den Herrschaftszeiten Cäsars und Caligulas. In einer bei Sueton für das Jahr 44 v. Chr. beschriebenen Szene versammelt Cäsar, zu jener Zeit dictator perpetuus des römischen Staates, den Senat auf dem forum Iulium ${ }^{5}$. Die Platzanlage war erst kurz zuvor aus eigenen Mitteln und zur Feier der eigenen Person und Familie fertiggestellt worden. Cäsar empfängt den Senat dort zur allgemeinen Empörung sitzend, pro aede Veneris Genetricis, vor dem Tempel der Venus Genetrix, wie Sueton ausdrücklich anmerkt. Dieser Ort ist vermutlich mit dem Podium des Tempels der Venus Genetrix zu identifizieren, in dem Venus als Ahnherrin des julischen Geschlechts verehrt wurde ${ }^{51}$. Vor dem in der Apsis des Tempels aufgestellten Bildnis der Venus und im Blickfeld der auf dem Platz versammelten Senatorenschaft hatte Cäsars Sitzenbleiben eine besondere Bedeutung. Cäsar inszenierte sich hier selbst als Kultbild. Er präsentierte sich zwar nicht ausdrücklich als Gott, spielte aber durch die bewusste Respektlosigkeit vor dem Senat höchst ambivalent mit den Ausdrucksmöglichkeiten römischer Repräsentation und den Näherungsstufen zwischen menschlicher und göttlicher Sphäre. Diese Inszenierung findet ihre Bühne und ihr Äquivalent in der Architektur des Platzes. Lässt sich Cäsars Empfang des Senats als ein Spiel mit Deutungen und Formen zeremoniellen Ausdrucks interpretieren, so ist eine für den Kaiser Caligula etwa 80 Jahre später überlieferte Szene weitaus weniger ambivalent. Über ihn berichten Sueton und Cassius Dio, dass er den Tempel der beiden Dioskuren, Castor und Pollux, auf dem Forum Romanum durch eine Brücke mit dem Palatin verband und ihn so zum Eingangsbereich des Kaiserpalastes umbaute. Er selbst soll zwischen den Statuen der Götter sitzend Hof gehalten haben ${ }^{52}$. Das war eine Steigerung der Götternähe Cäsars. Der Kaiser rückte ganz unmittelbar neben die Götterstatuen, wurde selbst zu einem lebenden Bildnis und verwandelte dabei das Forum Romanum in den Vorhof seines Domizils.

Beide Szenen belegen den Zusammenhang zwischen architektonischen Veränderungen und Handlungsformen und verweisen dabei insbesondere auf die Bedeutung, die in der römischen Kaiserzeit der baulich umgesetzten Nähe von Kaisern und Göttern zukam. Bereits eingangs wurde erwähnt, dass die Architektur des Maxentius repräsentative und zeremonielle Bedürfnisse dieser Herrschaft widerspiegelt. Für den Wiederaufbau des Venus- und Romatempels soll dies nun abschließend noch einmal genauer betrachtet werden. Die beiden Cellae des Venus- und Romatempels wichen in ihrer Neugestaltung unter Maxentius in bezeichnender Weise von der früheren hadrianischen Architektur

50

52

\footnotetext{
51 Vgl. Gros 1976, 134; Hölscher 2006, 193-194.

Suet.Iul. 78,1 .

Suet.Cal. 22; Cass.Dio 59, 28, 5 .
} 
$\mathrm{ab}^{53}$. An die Stelle der relativ schlichten älteren Cellae traten nun aufwendig mit seitlichen Statuennischen, Tonnengewölbe und Apsis geschmückte Prunksäle. Treppenanlagen scheinen die Apsispodien mit dem Fußbodenniveau der Cella verbunden zu haben und außergewöhnliche, illusionistisch gestaltete Apsiskalotten berücksichtigten unmittelbar die Blicke von Besuchern. Im neuen Venus- und Romatempel entstand unter Maxentius somit ein Mittelpunkt kaiserlicher Legitimation, wie er bis dahin nur in den Tempeln der Kaiserforen existiert hatte. Die Cellae orientierten sich an der Innenraumarchitektur anderer römischer Apsidentempel, wobei vor allem die Tempel des Cäsar- und des Augustusforums Vorbilder gewesen zu sein scheinen. Die in der Tempelarchitektur keineswegs häufig auftretende Form des Apsidentempels bezog ihre Bedeutung aus der klar strukturierten Trennung in Saal und Kultnische und damit in eine Menge von Personen auf der einen Seite und ein herausgehobenes Objekt auf der anderen ${ }^{54}$. Die Tempel der beiden ersten Kaiserforen hatten diese Raumform erstmals erprobt und standen dabei im Kontext der architektonischen Formensprache und ihrer ideellen Implikationen zu Beginn der Prinzipatszeit. Schon Pierre Gros hat diese Zusammenhänge hervorgehoben und mit der urbanistischen Gestaltung der Platzanlagen verknüpft ${ }^{55}$. Am Cäsarforum rückte das Götterbild an das Ende einer städtebaulichen Achse, die vom Platz über den Pronaos bis in die Cella hinein auf den Willen eines einzigen Bauherren zurückging. Venus war hier als Venus Genetrix für das julische Geschlecht okkupiert und ihre hierarchische Hervorhebung im Tempel unterstrich Cäsars Anspruch auf die führende Rolle im Staat ${ }^{56}$. Auch Cäsar war in Gestalt einer Panzerstatue schon zu Lebzeiten auf dem Platz präsent ${ }^{57}$ und ante Veneris Genetricis aedem war auch eine Statue seines Pferdes aufgestellt, die später wohl ebenfalls das Bildnis des verstorbenen Diktators trug ${ }^{5}$. Die Szene des Senatsempfangs durch den Diktator vor diesem Tempel illustriert insofern die architektonisch bereits vorgegebene Hierarchisierung der Platzanlage ${ }^{59}$. Das augusteische Platzprogramm des Augustusforums erweiterte und monumentalisierte diese Ansätze. Stärker noch als am Cäsarforum kulminiert in der Apsis des Mars-Ultor-Tempels ein dynastisches, ganz auf die Person des Bauherren ausgerichtetes und seine göttliche Abstammung thematisierendes Bildprogramm ${ }^{60}$. Wie auf dem Cäsarforum fanden auch hier Gerichtsver-

\footnotetext{
53 Ziemssen 2007, 74-82.

54 Gros 1976, 136-137.

55 Viele Tempelinnenräume wurden unter Augustus zu kostbar ausgestatteten, architektonisch vielfältigen Repräsentationssälen neu- oder umgestaltet. In der Nachfolge von Selbstdarstellungsformen der späten Republik erhielt die Repräsentation des einzelnen im Tempel seit Cäsar feste architektonische Form und die übergreifende Intention, den Kaiser durch seine Präsenz im Tempel in eine intime Nähe zu den Göttern zu rücken (Gros 1976, 155-169; Gros 1996, 154-159).

56 Zu Entstehung, Bedeutung und Nutzung des Forum Iulium vgl. Anderson 1984, 39-63.

57 Plin.nat. $34,18$.

58 Plin.nat. 8, 44, 155; Anderson 1984, 48.

59 Vgl. Gros 1976, 134.

60 Zanker 1972; Anderson 1984, 65-100.
} 
handlungen unter Vorsitz des Kaisers statt, siegreiche Generäle weihten die Abzeichen ihres Triumphes dem Mars Ultor, Statthalter zogen von hier aus in ihre Provinzen, Triumphatoren sollten auf dem Platz eine Bronzestatue erhalten, auch der Senat versammelte sich im Tempel und beschloss hier über Kriege und die Verleihung von Triumphen. Die Architektur mit dem Fluchtpunkt der Apsis bildete den Rahmen all dieser Zeremonien und ermöglichte die Übermittlung der Rolle des Princeps als dem einzigen Triumphator, Kriegsherrn und Lenker des Staates an das teilnehmende Volk ${ }^{61}$.

Da sich die architektonische Gestaltung der maxentianischen Cellae an diese alten Tempelvorgänger anlehnte, sollten auch die Ursachen dafür nicht nur im Wunsch nach der Wahl einer besonderen Aufwandsform gesehen werden ${ }^{62}$. Anzunehmen sind vielmehr ähnliche Nutzungsformen vor einem, wie wir sehen konnten, mit der frühen Kaiserzeit durchaus vergleichbaren ideologischen Hintergrund. Auch im templum urbis bot die Architektur nun den Rahmen für eine Inszenierung der Kultfiguren, von denen zumindest Roma in ähnlichem Nahverhältnis zum Kaiser stand wie einst Venus Genetrix zu Cäsar. Im hadrianischen Vorgängerbau waren solche Bezüge noch nicht vorhanden, daher hatten sie auch keine Umsetzung in der Architektur gefunden. Konkrete Funktionen im Herrscherzeremoniell wie für den Tempel des Mars Ultor oder Handlungsszenen wie jene vor dem Venus-Genetrix-Tempel sind für den Bau des Maxentius zwar nicht überliefert und denkbar ist angesichts der kurzen Regierungszeit des Herrschers auch, dass der Bau erst nach dessen Tod fertiggestellt wurde. Doch sind durchaus plausible Annahmen über die beabsichtigten Nutzungen möglich. Wie im Tempel des Mars Ultor könnten auch im Venus- und Romatempel Senatssitzungen abgehalten worden sein, wofür sich angesichts der speziellen Rolle der Roma für Maxentius deren Cella besonders anbot ${ }^{6_{3}}$. Der Kaiser selbst könnte - wie einst Cäsar - vor dem Tempel Versammlungen von Senat oder Volk präsidiert und sich dabei vor der in der Apsis thronenden Göttin als Herr des Staates inszeniert haben. Auf der Plattform außerhalb des Baues, aber auch im Tempelinneren ist die Aufstellung von Statuen des Maxentius und seiner Familienangehörigen anzunehmen, die im Zusammenhang mit solchen Staatsakten ähnliche Wirkung entfaltet hätten wie die Bildwerke der Kaiserforen. Tatsächlich könnte sich auch ein Überrest einer solchen Statuenaufstellung erhalten haben: Fundamentierungen vor den Apsispodien lassen sich möglicherweise wie im Mars-Ultor-Tempel zu einer Treppenanlage ergänzen, auf der Weihungen aufgestellt werden konnten. In der Westapsis hätte der dem Podium hier noch

61 Cass.Dio 55, 10, 2-5; Suet.Aug. 29, 1-2; Anderson 1984, 88-97.

62 Brandenburg 1992, 30. Ähnlich spricht zuletzt Oenbrink 2006, 181-184 vor allem von der »Pracht« der Räume und ihrer »zeittypischen Wandgliederung «.

63 Da wohl nur selten alle der in tetrarchischer Zeit ca. 6oo Senatoren gleichzeitig in Rom waren, spricht auch der begrenzte Platz in jeweils einer der Cellae nicht zwingend gegen eine solche Annahme. Eine unsichere Angabe in der Historia Augusta nennt für die Zeit des Alexander Severus eine vorgeschriebene Mindestzahl von 70 anwesenden Senatoren: SHA Alex. 16; vgl. zur realen Anwesenheit von Senatoren im spätantiken Rom Löhken 1982, 104-105. Zusammenfassend zu Tempeln als Sitzungsorten des Senats Hölscher 2006, 193. 
Abb. 8 | Maxentiusbasilika und Venus- und Romatempel im Modell des Museo della Civiltà Romana, Rom.

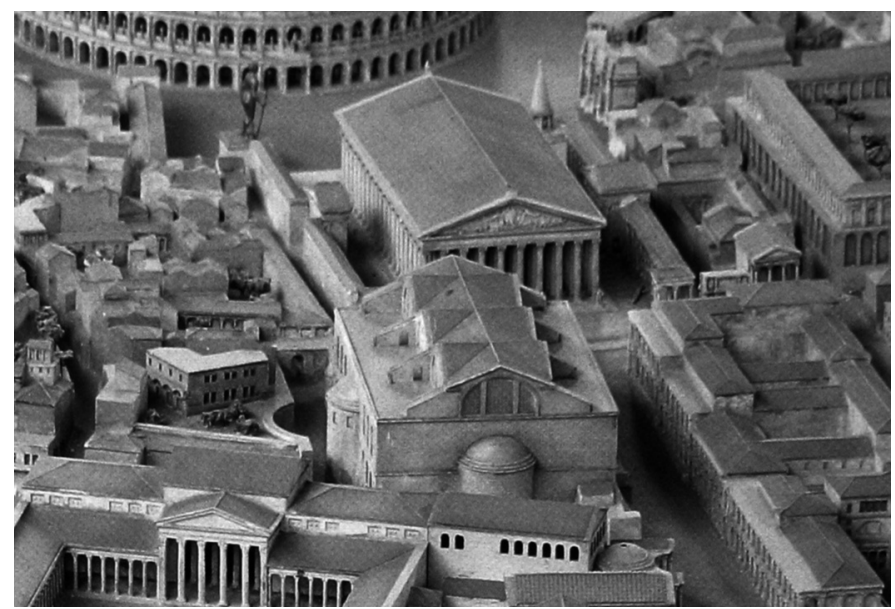

zusätzlich aufgesetzte Sockel sogar eine Anordnung der Statuen zugelassen, wie sie auf den Münzdarstellungen erscheint: Roma auf einem erhöhten Podest thronend, Maxentius etwas tiefer stehend ${ }^{64}$.

Bestandteil einer räumlichen Inszenierung war der Venus- und Romatempel aber auch in einem städtebaulichen Maßstab. Zwar bildete er nicht wie die Tempel der Kaiserforen den Abschluss einer geschlossenen Platzanlage, doch war die nach Westen ausgerichtete Cella ein Kulminationspunkte des Straßensystems zwischen Kaiserpalast und Forum Romanum, das die Herrscher seit dem 1. Jh. für die großen Umzüge des adventus, der profectio, der Leichenzüge zum Forum und der Auszüge zu den Spielen nutzten (Abb. 8) ${ }^{65}$. Dieses städtische Umfeld verwandelte sich unter Maxentius durch die Errichtung der Basilika. Unmittelbar gegenüber dem Tempel entstand mit diesem Neubau ein gewaltiger Empfangssaal des Kaisers, dessen Eingang sich demonstrativ nach Osten öffnete und dessen Apsis der westlichen Apsis des Venus- und Romatempels zugewandt war. In diesem neu angelegten städteplanerischen Bezugssystem sind nun auch Zeremonien zu erwarten, die das alte Spektrum kaiserlicher Umzüge ergänzten. Der Herrscher muss vom Palatin aus in die Basilika eingezogen sein, kann aber eine solche Prozession auch mit dem Opfer vor dem erneuerten Tempelbau verbunden haben. Die Bauten des Maxentius sind die Bühnen, auf denen der Herrscher als Schützling der Göttin Roma von den Untertanen gefeiert wurde.

In unmittelbarer Nähe des Venus- und Romatempels wurde vor wenigen Jahren auch eine der erstaunlichsten archäologischen Entdeckungen der letzten Zeit in Rom gemacht, die der Herrschaftsrepräsentation unter Maxentius einen entscheidenden Aspekt hinzu-

64 Vgl. dazu meine in Druckvorbereitung befindliche Dissertationsschrift.

65 Ziemssen 2010a. 


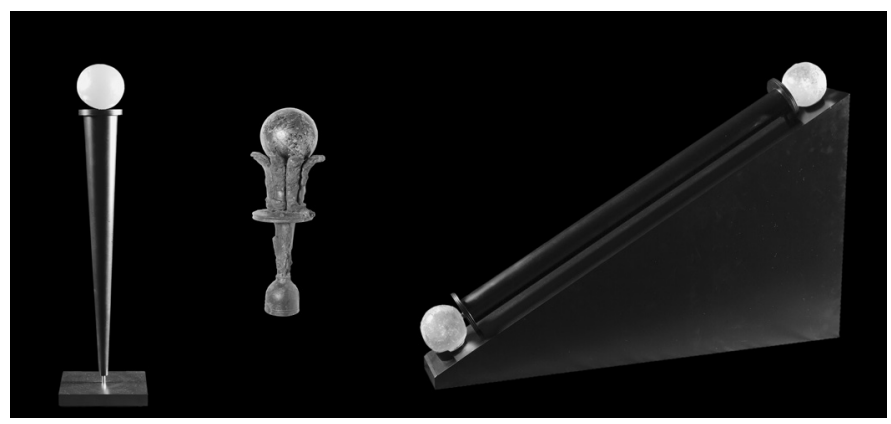

Abb. 9 | Die Szepter aus dem Fund am Nordhang des Palatin, von links nach rechts: konisches Szepter mit Globus aus Chalzedon; kleines Szepter mit Globus aus grünem Glas; Szepter mit zwei Globen aus vergoldetem Glas.

fügen $\mathrm{kann}^{66}$. In einem Raum an der südlichen Seite der zum Titusbogen ansteigenden Straße, der zu einem größeren Baukomplex unklarer Funktion gehörte, wurde das in der Antike angelegte Versteck einer Reihe zeremoniell genutzter Gegenstände gefunden (Abb. 9): ein Kurzszepter mit Glasglobus, Reste eines längeren Szepters mit je einem Glasglobus an jeder Seite, ein weiterer einem dritten Szepter zugehöriger Globus sowie die Reste von Paradelanzen und Standarten, alles in Seidentuch gewickelt und bewusst vergraben. Es sind, daran kann kein Zweifel bestehen, kaiserliche Insignien. Die Ausgräber haben den Zeitpunkt des Versteckens auf Basis der Stratigraphie zwischen den Beginn des 3. und den Beginn des 4. Jhs. datiert. Eine Reihe historischer Erwägungen, ergänzt um die Art und Weise der Deponierung, führt sie schließlich zur weitergehenden und überzeugenden Hypothese, es habe sich um die Herrschaftszeichen des Maxentius gehandelt, die im Zusammenhang des Kampfes gegen Konstantin vor dem Sieger in Sicherheit gebracht werden sollten ${ }^{67}$. Doch auch ohne diese Datierung zu akzeptieren, bezeugt der Fund in eindrucksvoller Weise die Realität von Gegenständen, die im Bild zunächst als bloße Symbole erscheinen mögen, und die Existenz zeremonieller Handlungen im Umfeld des Palatin.

\section{Schlussbemerkungen: Bild, Raum und Handlung im maxentianischen Rom}

Die Darstellungen der maxentianischen Münzen mit ihrem eigentümlichen Wechsel zwischen Realität und Symbolik bildeten den Ausgangspunkt der Untersuchung. Sie lassen sich, wie wir sehen konnten, als Ausdruck dauerhafter, göttlich fundierter Herrschaft deuten und standen damit sowohl inhaltlich als auch formal in enger Entsprechung zum Vorgehen der lateinischen Panegyriker, die reales Geschehen beschrieben, es aber zugleich auch ideologisch bewerteten. Der vorliegende Aufsatz legte den Fokus auf die Wir-

66 Panella et al. 2008; Panella 2011.

67 Panella et al. 2008, 716-718. 
kungsweise der Bilder und auf deren reziproken Bezug zu den Handlungsformen ihrer Zeit. Dabei ist der Unterschied dieser Darstellungen zu allen anderen im Band »Bild Raum - Handlung« analysierten Bild-Kontexten deutlich und ergibt sich aus der Natur des Mediums selbst. Die Münzbilder begleiteten das Geschehen im Rom des Maxentius nicht in derselben Art und Weise wie dies die Fresken im Palast von Knossos oder auf den Platzanlagen von Karkemish im Fall ihrer jeweiligen Zeremonien taten; sie standen den Teilnehmern der zeremoniellen Handlungen eben nicht am Ort des Geschehens selbst und nur dort - als örtlich fixierte Monumente vor Augen. Gleichwohl lautet die Prämisse unseres Bandes, dass räumliche Zusammenhänge stets das Ergebnis von Konstruktionen der beteiligten Personen sind. In diesem Sinne eröffneten die follis-Prägungen ein Wechselspiel mit den realen Vorgängen in Rom. Die Betrachter verbanden beim Blick auf die Münze die ikonographischen Angaben mit dem Wissen, das sie aus eigener Anschauung oder aus Berichten vom Umfeld des Tempels der Venus und Roma hatten; umgekehrt rief der Anblick der Tempel-Baustelle und der dort stattfindenden Zeremonien bei den Zeitgenossen zwangsläufig das symbolisch verdichtete Abbild dieses Ortes auf den Münzen wach. Die maxentianischen Münzen stellen uns damit einem medialen Phänomen gegenüber, das der gesamten antiken Münzprägung eigen ist und diese eng mit der uns so vertrauten Bilderwelt des cyberspace verwandt sein lässt. Gerade die in der Bronzeprägung massenhaft verbreiteten Motive gewannen eine Wirksamkeit, die weder über den Ort der Darstellung noch über den der Betrachtung hinreichend zu erfassen ist. Der Begriff »Handlung« wird dabei zu einem heuristischen Schlüsselbegriff, denn es ist gerade das im Münzbild dargestellte Handlungsgeschehen, das in seiner klaren räumlichen Bestimmung auf eine zeremonielle Realität verwies. Erst über diesen eindeutigen Bezug wurde das Bild wirksam und entfaltete seine Aussagekraft. Eine isolierte, ideologiegeschichtliche Deutung der Bilder greift daher zu kurz. Wenn auch die Literatur in Gestalt der Panegyrici ihre Herrschaftssymbolik in ähnlicher Weise zu konkretisieren versuchte - wir konnten dies am Beispiel von Konstantins Besuch im Apollon-Tempel sehen -, waren doch die Bilder der Münzen von einzigartiger Wirksamkeit. Nur in ihnen wurden die komplexen Handlungsvollzüge im Rom des beginnenden 4. Jh. und deren ideologische Hintergründe mit einem einzigen Blick sichtbar, nachvollziehbar und mit Sinn erfüllt.

\section{Bibliographie}

Alföldi I97I

A. Alföldi, Der Vater des Vaterlandes im römischen Denken (Darmstadt 1971).

Anderson 1984

J. C. Anderson, The Historical Topography of the Imperial Fora (Brüssel 1984). 


\section{Barceló 2007}

P. Barceló, Constantins Visionen: Zwischen Apollo und Christus, in: P. Barceló -

V. Rosenberger (Hgg.), Humanitas - Beiträge zur antiken Kulturgeschichte. Festschrift Gunther Gottlieb (München 2001) 45-61.

\section{Beaujeu 1955}

J. Beaujeu, La Religion romaine à l'apogée de l'empire. I: La Politique religieuse des Antonins (Paris 1955).

\section{Benjamin 1963}

A. S. Benjamin, The Altars of Hadrian in Athens and Hadrian's Panhellenic Program, Hesperia $32,1963,57-86$.

\section{Bernand 1977}

A. Bernand, Pan du désert (Leiden 1977).

\section{Bernand 1984}

E. Bernand, Epigraphie grecque et architecture égyptienne à l'époque imperiale, in:

H. Walter (Hg.), Hommages à Lucien Lerat (Paris 1984) 73-89.

\section{Bourdieu 1974}

P. Bourdieu, Zur Soziologie der symbolischen Formen (Frankfurt a. M. 1974).

\section{Brandenburg 1992}

H. Brandenburg, Die konstantinischen Kirchen in Rom. Staatstragender Kult und Herrscherkult zwischen Tradition und Neuerung, in: O. Brehm - S. Klie (Hgg.), Musikos aner, Festschrift M. Wegner (Bonn 1992) 27-58.

\section{Carson 1980}

R. A. G. Carson, A Treasure of Aurei and Multiples from the Mediterranean, in:

P. Bastien - F. Dumas - H. Huvelin - C. Morrisson (Hgg.), Mélanges de numismatique d'archéologie et d'histoire, Festschrift Jean Lafaurie (Paris 1980) 59-74.

\section{Casey 1977}

P. J. Casey, Tradition and Innovation in the Coinage of Carausius and Allectus, in:

J. Munby - M. Henig (Hgg.), Roman Life and Art in Britain. A Celebration in Honour of the Eightieth Birthday of Jocelyn Toynbee, BAR 41 (Oxford 1977) 217-230.

\section{Casey 1994}

P. J. Casey, Carausius and Allectus. The British Usurpers (London 1994). 


\section{Christern 1986}

J. Christern, Die >Gerichtsbasilika < beim Forum von Tipasa (Neuaufnahme), ihre Funktion und die Frage nach den Vorbildern für den basilikalen Kirchenbau, in:

O. Feld et al. (Hg.), Studien zur spätantiken und byzantinischen Kunst 1, Festschrift Friedrich Wilhelm Deichmann (München 1986) 163-204.

\section{Cullhed 1994}

M. Cullhed, Conservator Urbis Suae: Studies in the Politics and Propaganda of the Emperor Maxentius (Stockholm 1994).

\section{Daguet-Gagey 2004}

A. Daguet-Gagey, Septime Sévère et ses fils, Restitutores Urbis, La personnalisation des mérites impériaux, RNum 160, 2004, 175-199.

\section{Desnier 1993}

J. L. Desnier, Omina et Realia. Naissance de l'Urbs sacra sévérienne (193-204 ap. J.-C.), MEFRA 105 (2), 1993, 547-620.

\section{Fears 1977}

J. R. Fears, Princeps a diis electus. The Divine Election of the Emperor as a Political Concept at Rome (Rom 1977).

\section{Flaig 1992}

E. Flaig, Den Kaiser herausfordern. Die Usurpation im Römischen Reich (Frankfurt a M. 1992).

\section{Gagé 1936}

J. Gagé, Le Templum Urbis et les origines de l'idée de « renovatio », in: Mélanges Franz Cumont, AIPhOr 4 (Bruxelles 1936) 151-187.

\section{Gros 1976}

P. Gros, Aurea Templa. Recherches sur l'architecture religieuse de Rome à l'époque d'Auguste, BEFAR 231 (Rom 1976).

\section{Gros 1996}

P. Gros, Larchitettura romana. Dagli inizi del III secolo a.C. alla fine dell'alto impero. I monumenti pubblici (Mailand 1996).

\section{Guarducci 1974}

M. Guarducci, Epigrafia Greca Bd. 3 (Roma 1974).

\section{Handler I97I}

S. Handler, Architecture on the Roman Coins of Alexandria, AJA 75, 1971, 57-74. 


\section{Hänlein-Schäfer 1985}

H. Hänlein-Schäfer, Veneratio Augusti. Eine Studie zu den Tempeln des ersten römischen Kaisers (Rom 1985).

\section{Hausmann 200I}

C. Hausmann, Die Wechselfälle des Templum Veneris et Romae von der Antike bis zur Renaissance, in: S. Bruzzi - D. Käch - E. Kistler et al. (Hgg.), Zona Archeologica. Festschrift Hans Peter Isler (Bonn 2001) 193-204.

\section{Hill 1964}

P. V. Hill, The Coinage of Septimius Severus and His Family of the Mint of Rome, A.D. 193-217 (London 1964).

\section{Hölscher 1967}

T. Hölscher, Victoria Romana. Archäologische Untersuchungen zur Geschichte und Wesensart der römischen Siegesgöttin von den Anfängen bis zum Ende des 3. Jhs. n. Chr. (Mainz 1967).

\section{Hölscher 1980}

T. Hölscher, Die Geschichtsauffassung in der römischen Repräsentationskunst, JdI 95, $1980,265-321$.

\section{Hölscher 2006}

T. Hölscher, Macht, Raum und visuelle Wirkung. Auftritte römischer Kaiser in der Staatsarchitektur von Rom, in: J. Maran - C. Juwig - H. Schwengel - U. Thaler (Hgg.), Constructing Power. Architecture, Ideology and Social Practice. Konstruktion der Macht. Architektur, Ideologie und soziales Handeln (2006) 185-205.

\section{Hornbostel 1973}

W. Hornbostel, Sarapis. Studien zur Überlieferungsgeschichte, den Erscheinungsformen und Wandlungen der Gestalt eines Gottes (Leiden 1973).

\section{Jelocnik 1973}

A. Jelocnik, Centurska zakladna najdba - The Centur Hoard (Ljubljana 1973).

\section{Kolb 200I}

F. Kolb, Herrscherideologie in der Spätantike (Berlin 2001).

\section{Körner 2002}

C. Körner, Philippus Arabs. Ein Soldatenkaiser in der Tradition des antoninischseverischen Prinzipats, Untersuchungen zur antiken Literatur und Geschichte $\sigma_{1}$ (Berlin 2002). 


\section{Küthmann I973}

B. H. Küthmann - D. Overbeck - I. Weber Steinhilber, Bauten Roms auf Münzen und Medaillen (München 1973).

\section{Lehnen 1997}

J. Lehnen, Adventus Principis. Untersuchungen zu Sinngehalt und Zeremoniell der Kaiserankunft in den Städten des Imperium Romanum, Prismata. Beiträge zur Altertumswissenschaft 7 (Frankfurt a. M. 1997).

\section{Löhken 1982}

H. Löhken, Ordines dignitatum. Untersuchungen zur formalen Konstituierung der spätantiken Führungsschicht, Kölner historische Abhandlungen 30 (Köln 1982).

\section{MacCormack I98I}

S. G. MacCormack, Art and Ceremony in Late Antiquity (Berkeley 1981).

\section{Martin I984}

J. Martin, Zum Selbstverständnis, zur Repräsentation und Macht des Kaisers in der Spätantike, Saeculum 35, 1984, 115-131.

McKenzie - Gibson - Reyes 2004

J. S. McKenzie - S. Gibson - A. T. Reyes, Reconstructing the Serapeum in Alexandria from the Archaeological Evidence, JRS 94, 73-121.

\section{Milne 1933}

J. G. Milne, Catalogue of Alexandrian Coins (Oxford 1933).

\section{Nixon - Rodgers 1994}

C. E. V. Nixon - B. S. Rodgers, In Praise of Later Roman Emperors. The Panegyrici Latini. Introduction, Translation, and Historical Commentary with the Latin Text of R.A.B. Mynors (Berkeley 1994).

\section{Oenbrink 2006}

W. Oenbrink, Maxentius als conservator urbis suae. Ein antitetrarchisches Herrschaftskonzept tetrarchischer Zeit, in: D. Boschung - W. Eck (Hgg.), Die Tetrarchie. Ein neues Regierungssystem und seine mediale Präsentation, Kolloquium des Lehr- und Forschungszentrums für die Antiken Kulturen des Mittelmeerraumes der Universität zu Köln, 13.-14. Februar 2004 (Köln 2006) 168-209.

\section{Panella et al. 2008}

C. Panella - A. F. Ferrandes - G. Pardini - M. Ricci, Le insegne imperiali dal Palatino, ScAnt 13, 2006 (2008), 715-740. 


\section{Panella 20II}

C. Panella, I Segni del potere. Realtà e immaginario della sovranità nella Roma imperiale (Bari 2011).

Price - Trell 1977

M. J. Price - B. L. Trell, Coins and Their Cities. Architecture on the Ancient Coins of Greece, Rome and Palestine (London 1977).

Radt 1999

W. Radt, Pergamon. Geschichte und Bauten einer antiken Metropole (Darmstadt 1999).

\section{Rodgers I980}

B. S. Rodgers, Constantine's Pagan Vision, Byzantion 50, 1980, 259-278.

\section{Scott Ryberg 1955}

I. Scott Ryberg, Rites of the State Religion in Roman Art (Rome 1955).

\section{Shiel 1977}

S. Shiel, The Episode of Carausius and Allectus: the Literary and Numismatic Evidence, BAR 40 (Oxford 1977).

\section{Toynbee 1986}

J. M. C. Roman Medaillons. With an Introduction to the Reprint Edition (New York 1986).

\section{Tran tam Tinh 1983}

V. Tran tam Tinh, Sérapis debout. Corpus des monuments de Sérapis debout et étude iconographique (Leiden 1983).

\section{Tran tam Tinh 1996}

V. Tran tam Tinh, Les empereurs romains versus Isis, Sérapis, in: A. Small (Hg.), Subject and Ruler: The Cult of the Ruling Power in Classical Antiquity (Ann Arbor 1996) 215-230.

\section{Vogt 1924}

J. Vogt, Die alexandrinischen Münzen. Grundlegung einer alexandrinischen Kaisergeschichte (Stuttgart 1924).

\section{Willers I990}

D. Willers, Hadrians panhellenisches Programm. Archäologische Beiträge zur Neugestaltung Athens durch Hadrian (Bern 1990).

\section{Zanker 1972}

P. Zanker, Forum Augustum (Tübingen 1972).

\section{Zanker 1997}

P. Zanker, In Search of the Roman Viewer, in: D. Buitron-Oliver (Hg.), The Interpretation of Architectural Sculpture in Greece and Rome (Washington, D.C. 1997) 178-191. 


\section{Ziemssen 2007}

H. Ziemssen, Maxentius und Rom - Das neue Bild der Ewigen Stadt, in: H. Leppin -

H. Ziemssen, Maxentius. Der letzte Kaiser in Rom (Mainz 2007) 35-122.

\section{Ziemssen 20I0a}

H. Ziemssen, Palast und städtischer Raum. Wandel und Kontinuität des römischen Stadtzentrums (1.-4. Jh. n. Chr.), Hephaistos 26, 2008 (2010), 177-191.

\section{Ziemssen 20Iob}

H. Ziemssen, Roma Auctrix Augusti. Die Veränderungen des römischen Stadtbilds unter Kaiser Maxentius (306-312 n.Chr.), in: N. Burkhardt - R. H. W. Stichel (Hgg.), Die antike Stadt im Umbruch. Kolloquium in Darmstadt, 19. bis 20. Mai 2006 (Wiesbaden 2010) 16-27.

\section{Ziemssen 2012}

H. Ziemssen, Die Kaiserresidenz Rom in der Zeit der Tetrarchie, in: T. Fuhrer (Hg.), Rom und Mailand in der Spätantike. Repräsentationen städtischer Räume in Literatur, Architektur und Kunst, Topoi. Berlin Studies of the Ancient World 4 (Berlin 2012) 87-110.

\section{Abbildungsnachweis}

Abb. 1: Kent/Overbeck/Stüler/Hirmer, Die römische Münze, Nr. 619, Taf. 35; Abb. 2: Photographie H. Ziemssen, Münzarchiv Eisenlohr; Abb. 3: Image courtesy of Classical Numismatic Group; Abb. 4: Image courtesy of Roma Numismatics Ltd., Auction 3, lot nr. 503; Abb. 5: nach Milne 1933 Taf. 4 Nr. 1380; Abb. 6: Numismatische Bilddatenbank Eichstätt; Abb. 7. 8: Photographie H. Ziemssen; Abb. 9: Szepter mit Globus aus Chalzedon Photographie L. Mandato und G. Cargnel, Negativ SSBAR 551157; Szepter mit Globus aus grünem Glas Photographie M. Necci; Szepter mit zwei Globen aus vergoldetem Glas Photographie L. Mandato und G. Cargnel, Negativ SSBAR 551159. 
